\title{
The photon dominated region in the IC 348 molecular cloud
}

\author{
K. Sun ${ }^{1}$, V. Ossenkopf ${ }^{1,2}$, C. Kramer ${ }^{1}$, B. Mookerjea ${ }^{3}$, M. Röllig $^{4}$, M. Cubick ${ }^{1}$, and J. Stutzki ${ }^{1}$ \\ 1 I. Physikalisches Institut, Universität zu Köln, Zülpicher Straße 77, 50937 Köln, Germany \\ e-mail: kefeng@ph1.uni-koeln.de \\ 2 SRON Netherlands Institut for Space Research, PO Box 800, 9700 AV Groningen, The Netherlands \\ 3 Department of Astronomy \& Astrophysics, Tata Institute of Fundamental Research, Homi Bhabha Road, Mumbai 400 005, India \\ 4 Argelander-Institut für Astronomie, Universität Bonn, Auf dem Hügel 71, 53121 Bonn, Germany
}

Received 7 August 2007 / Accepted 17 July 2008

\section{ABSTRACT}

\begin{abstract}
Aims. In this paper we discuss the physical conditions of clumpy nature in the IC 348 molecular cloud. We show that the millimeter and sub-millimeter line emission from the IC 348 molecular cloud can be modelled as originating from a photon dominated region (PDR).

Methods. We combine new observations of fully sampled maps in [CI] at $492 \mathrm{GHz}$ and ${ }^{12} \mathrm{CO} 4-3$, taken with the KOSMA $3 \mathrm{~m}$ telescope at about $1^{\prime}$ resolution, with FCRAO data of ${ }^{12} \mathrm{CO} 1-0,{ }^{13} \mathrm{CO} 1-0$ and far-infrared continuum data observed by HIRES/IRAS. To derive the physical parameters of the region we analyze the line ratios of $[\mathrm{CI}]{ }^{3} \mathrm{P}_{1}-{ }^{3} \mathrm{P}_{0} /{ }^{12} \mathrm{CO} 4-3$, $\left.[\mathrm{CI}]\right]^{3} \mathrm{P}_{1}-{ }^{3} \mathrm{P}_{0} /{ }^{13} \mathrm{CO} 1-0$, and ${ }^{12} \mathrm{CO} 4-3 /{ }^{12} \mathrm{CO} 1-0$. A first rough estimate of abundance is obtained from an LTE analysis. To understand the [C I] and $\mathrm{CO}$ emission from the PDRs in IC 348, we use a clumpy PDR model. With an ensemble of identical clumps, we constrain the total mass from the observed absolute intensities. Then we apply a more realistic clump distribution model with a power law index of 1.8 for clump-mass spectrum and a power law index of 2.3 for mass-size relation.

Results. We provide detailed fits to observations at seven representative positions in the cloud, revealing clump densities between $4 \times 10^{4} \mathrm{~cm}^{-3}$ and $4 \times 10^{5} \mathrm{~cm}^{-3}$ and C/CO column density ratios between 0.02 and 0.26 . The derived FUV flux from the model fit is consistent with the field calculated from FIR continuum data, varying between 2 and 100 Draine units across the cloud. We find that both an ensemble of identical clumps and an ensemble with a power law clump mass distribution produce line intensities which are in good agreement (within a factor $\sim 2$ ) with the observed intensities. The models confirm the anti-correlation between the $\mathrm{C} / \mathrm{CO}$ abundance ratio and the hydrogen column density found in many regions.
\end{abstract}

Key words. ISM: clouds - ISM: dust, extinction - ISM: structure - ISM: individual objects: IC 348

\section{Introduction}

Photon Dominated Regions (PDRs) are surfaces of molecular clouds where chemistry and heating are regulated by farultraviolet (FUV) photons $(6.0 \mathrm{eV}<h v<13.6 \mathrm{eV}$ ) (Hollenbach $\&$ Tielens 1999). Gas cooling happens mainly via the fine structure lines of $[\mathrm{OI}],[\mathrm{CII}],[\mathrm{CI}]$ and the rotational lines of $\mathrm{CO}$ (Kaufman et al. 1999). The form of carbon changes with increasing depth from the surface of the PDR from $\mathrm{C}^{+}$through $\mathrm{C}^{0}$ to $\mathrm{CO}$. Therefore emission from [C II], [C I] and the rotational lines of $\mathrm{CO}$ can be used as probes of temperature, density and column density in the PDRs (Kramer et al. 2004; Mookerjea et al. 2006). In particular, the two fine structure lines of neutral atomic carbon at 492 and $809 \mathrm{GHz}$ give information on intermediate regions between atomic and molecular gas, since they trace both physical regimes. Initial homogeneous plane-parallel steadystate models of PDRs predicted that PDRs are confined to the FUV irradiated surfaces of molecular clouds. However, observations detected widespread [C II] and [C I] emission, thus suggesting that PDR surfaces are formed deeply inside the molecular clouds. One possible explanation is in terms of the clumpiness of the molecular clouds which allows for deeper penetration of FUV radiation so that PDRs are formed at the surfaces of the clumps present throughout the molecular cloud (Stutzki et al. 1988; Howe et al. 1991; Mookerjea et al. 2006). In addition, an explanation has also been proposed in terms of the dynamics of the ISM: a. clouds are suddenly shielded from FUV fields
(Störzer et al. 1997); b. time-dependent chemistry of the PDRs (Oka et al. 2004)

IC 348 is a young open cluster northeast of the Perseus cloud at a distance of $316 \mathrm{pc}$ (Herbig 1998). The IC 348 cluster has about 300 stellar members, with spectral types from B5 to late $\mathrm{M}$, as found from observations in X-rays (Preibisch et al. 1996; Preibisch \& Zinnecker 2001), near-infrared (Lada \& Lada 1995; Luhman et al. 1998; Najita et al. 2000; Luhman et al. 2005), and optical (Herbig 1998; Luhman et al. 2003). Lada et al. (2006) constructed optical-infrared spectral energy distributions (SEDs) for all known members of the cluster by combining the Spitzer Space Telescope and ground-based observations. The IC 348 molecular cloud prominently shows up in the CO 3-2 survey of the Perseus molecular cloud complex (Sun et al. 2006). The FUV radiation of its youngest member, HD 281159, a B 5 V star, heats the surrounding gas and dust. Surveys for molecular outflows revealed so far four bipolar outflows powered by deeply embedded young sources (Eislöffel et al. 2003; Tafalla et al. 2006). Jørgensen et al. (2007) estimated the current star formation efficiency of $10 \%-15 \%$ for the whole Perseus cloud using Spitzer mid-infrared data and SCUBA sub-millimeter maps.

Sun et al. (2006) observed IC 348 in four low-J CO transitions as part of their 7.1 square degree survey of the entire Perseus molecular cloud and quantified the spatial structure of the emission in terms of its power spectrum, applying the $\Delta$-variance method (Stutzki et al. 1998) to the integrated 
intensity maps. The $\Delta$-variance spectra of the overall region was found to follow a power law with an index of $\beta=2.9-3.0$; while sub-regions of Perseus gave different power spectral indices. The active star forming regions showed higher indices typical for large condensations; lower indices were derived for dark clouds, representing more filamentary structure. IC 348 as an exception, showed a rather filamentary structure corresponding to the smallest value of $\beta=2.71$ in ${ }^{13} \mathrm{CO} 2-1$.

In this paper we present large-scale $\left(20^{\prime} \times 20^{\prime}\right)$ fully-sampled maps of the $[\mathrm{CI}]{ }^{3} \mathrm{P}_{1}-{ }^{3} \mathrm{P}_{0}$ (hereafter [CI]) and ${ }^{12} \mathrm{CO} 4-3$ emission from the IC 348 molecular cloud. At a common resolution of $70^{\prime \prime}$, we have combined our data with the ${ }^{12} \mathrm{CO} 1-0,{ }^{13} \mathrm{CO}$ 1-0 data from the Five College Radio Astronomy Observatory (FCRAO) (Ridge et al. 2003), and the far-infrared (FIR) continuum data from HIRES/IRAS. The goal of the analysis is to understand the extent to which the observed line emission from IC 348 can be understood in the framework of a photon dominated region.

Section 2 describes the KOSMA observations and the complementary datasets used for analysis. Section 3 discusses the general observational results. A Local Thermodynamic Equilibrium (LTE) analysis of the observed line intensities is presented in Sect. 4. A comparison with clumpy PDR models is presented in Sect. 5. Section 6 summarizes the results.

\section{Datasets}

\section{1. [ $\mathrm{Cl}$ ] and ${ }^{12} \mathrm{CO} 4-3$ observations with KOSMA}

We have used the Kölner Observatorium für Sub-Millimeter Astronomie (KOSMA) 3-m sub-millimeter telescope on Gornergrat, Switzerland (Winnewisser et al. 1986; Kramer et al. 1998a) to observe the emission of the fine structure line of neutral carbon at $492 \mathrm{GHz}$ and ${ }^{12} \mathrm{CO} 4-3$ at $461 \mathrm{GHz}$. We have used the Sub-Millimeter Array Receiver for Two frequencies (SMART) on KOSMA for these observations (Graf et al. 2002). SMART is an eight-pixel dual-frequency SIS-heterodyne receiver capable of observing in the 650 and $350 \mu \mathrm{m}$ atmospheric windows (Graf et al. 2002). The IF signals were analyzed with two $4 \times 1 \mathrm{GHz}$ array-acousto-optical spectrometers with a spectral resolution of $1.5 \mathrm{MHz}$ (Horn et al. 1999). The typical double side band receiver noise temperature at $492 \mathrm{GHz}$ is about $150 \mathrm{~K}$. The observations were performed in position-switched On-TheFly (OTF) between December 2004 and February 2005. Owing to technical difficulties the higher frequency channel of SMART could not be used at the time of these observations.

We observed a fully sampled map of the IC 348 molecular cloud centered at $\alpha=03^{\mathrm{h}} 44^{\mathrm{m}} 10^{\mathrm{s}}, \delta=32^{\circ} 06^{\prime}(\mathrm{J} 2000)$, extending over $20^{\prime} \times 20^{\prime}$. For the observations, we used the positionswitched on-the-fly (OTF) mode (Beuther et al. 2000) with a sampling of $29^{\prime \prime}$. The emission-free off position was selected from the ${ }^{13} \mathrm{CO} 1-0$ FCRAO map, which is $\left(-8^{\prime}, 10^{\prime}\right)$ relative to the map center. We estimate the pointing accuracy to be better than $10^{\prime \prime}$. The half power beam width (HPBW) and the main beam efficiency $\left(B_{\text {eff }}\right)$ were derived from continuum scans of the Sun and the Jupiter. The HPBW at both frequencies is $60^{\prime \prime}$ and the main beam efficiency is $50 \%$. The forward efficiency $F_{\text {eff }}$ of the telescope is $\sim 90 \%$. Atmospheric calibration was done by measuring the atmospheric emission at the OFF-position and using a standard atmospheric model to fit the opacity taking into account the sideband imbalance (Cernicharo 1985; Hiyama 1998).

All data presented in this paper are in units of main beam temperature $T_{\mathrm{mb}}$, calculated from the observed calibrated antenna temperature $T_{\mathrm{A}}^{*}$ using the derived beam and forward efficiencies, $T_{\mathrm{mb}}=T_{\mathrm{A}}^{*}\left(F_{\mathrm{eff}} / B_{\text {eff }}\right)$. Based on observations of reference sources such as W 3 and DR 21 we estimate the accuracy of the absolute intensity calibration to be better than $15 \%$. The data reduction was carried out using the GILDAS ${ }^{1}$ software package.

\subsection{Complementary data sets}

We have used the FCRAO ${ }^{12} \mathrm{CO} 1-0$ and ${ }^{13} \mathrm{CO} 1-0$ datasets with an resolution of 46" presented by Ridge et al. (2003) for comparison. For comparison, we have smoothed all $\mathrm{CO}$ and $\mathrm{C}$ I data to a common resolution of $70^{\prime \prime}$ assuming that both the FCRAO and KOSMA telescopes have a Gaussian beam.

Further, we have obtained HIRES processed IRAS maps of the dust continuum emission at 60 and $100 \mu \mathrm{m}$ (Aumann et al. 1990). The dust continuum maps have resolution of 1.5 , which is only slightly worse than that of our submillimeter datasets.

\section{Observational results}

Figure 1 presents maps of integrated intensities of [C I $],{ }^{12} \mathrm{CO}$ $1-0,{ }^{13} \mathrm{CO} 1-0$ and ${ }^{12} \mathrm{CO} 4-3$ observed in IC 348 . All maps are integrated over the velocity range $V_{\mathrm{LSR}}=2 \mathrm{~km} \mathrm{~s}^{-1}$ to $14 \mathrm{~km} \mathrm{~s}^{-1}$.

The energy of the upper level for the [C I] 1-0 transition is $24 \mathrm{~K}$ and the critical density for this transition is $10^{3} \mathrm{~cm}^{-3}$ for collisions with molecular $\mathrm{H}_{2}$ (Schröder et al. 1991). This implies that [CI] 1-0 is easily excited and the line is easily detectable even when emitted by moderate density interstellar gas exposed to the average interstellar radiation field. Previous observations have found the $[\mathrm{CI}$ to be extended and well correlated with the low-J CO emission (see a review by Preibisch et al. 1996; Schneider et al. 2003; Mookerjea et al. 2006). In IC 348 the [C I] emission peaks to the southwest of the mapped region with a value of $24 \mathrm{~K} \mathrm{~km} \mathrm{~s}^{-1}$ and we detect [C I] emission over a large region with a homogeneous intensity distribution at a level of $65 \%$ of the peak intensity. The [C I] emission is extended towards the east and northeast of the mapped region. This is consistent with the clumpy UV irradiated cloud scenario.

The ${ }^{12} \mathrm{CO} 4-3$ emission peaks at almost the same position as [C I] with an intensity of $\sim 66 \mathrm{~K} \mathrm{~km} \mathrm{~s}^{-1}$. However, the morphology of the ${ }^{12} \mathrm{CO} 4-3$ emission is different to that of [C I]. We find secondary maxima in the north and a steeper decay of the emission to the south. The distribution of the ${ }^{12} \mathrm{CO} 4-3$ emission agrees very well with that of ${ }^{12} \mathrm{CO} 3-2$ (Sun et al. 2006). We attribute the difference in the intensity distributions of [C $\mathrm{I}]$ and ${ }^{12} \mathrm{CO} 4-3$ to the fact that ${ }^{12} \mathrm{CO} 4-3$ emission arises from regions of higher temperature and density, while [C I] emission can arise also from embedded PDR surfaces with moderate to low density within the molecular clouds.

The distribution of the ${ }^{12} \mathrm{CO} 1-0$ emission shows the same global structure as the ${ }^{12} \mathrm{CO} 4-3$ emission, but it is considerably more extended. This is consistent with the fact that ${ }^{12} \mathrm{CO} 1-0$ traces lower temperature and density. The morphologies of [C I] and ${ }^{13} \mathrm{CO} 1-0$ appear to be very similar, implying that they trace the same material and both lines are effectively column density tracers.

We selected seven positions within IC 348 for a more detailed study. Six of the seven positions are oriented along a cut from the northern edge of the cloud, past HD 281159 and into the clouds. The seventh position is at the southeast edge of the cloud. Though emission at the seventh position (southern rim) is weak, it is clearly detected. The selected positions vary in their

\footnotetext{
${ }^{1}$ http://www.iram.fr/IRAMFR/GILDAS/
} 

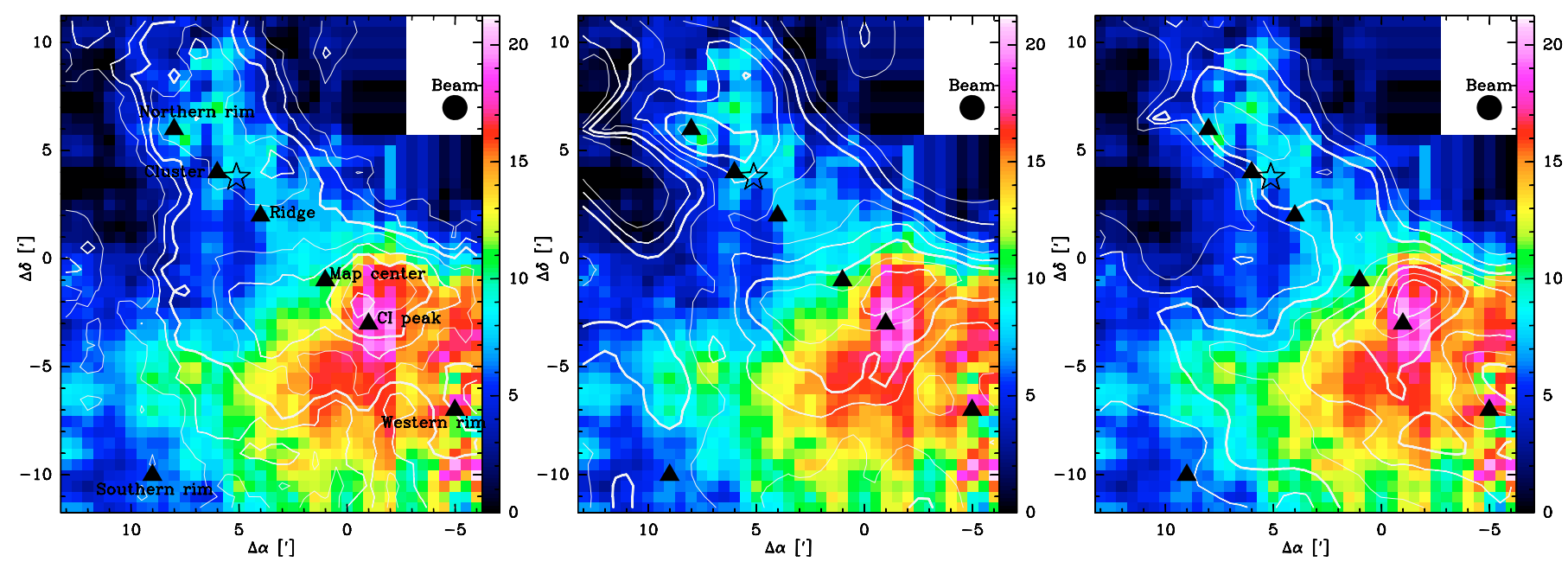

Fig. 1. Velocity integrated intensities of [CI] emission (color) overlayed with contours of a) ${ }^{12} \mathrm{CO} 4-3$, b) ${ }^{12} \mathrm{CO} 1-0$ and c) ${ }^{13} \mathrm{CO} 1-0$ integrated intensities at a common angular resolution of $70^{\prime \prime}$. The center of the maps is at $\alpha=03^{\mathrm{h}} 44^{\mathrm{m}} 10^{\mathrm{s}}, \delta=32^{\circ} 06^{\prime}$ (J2000). All tracers are integrated from $V_{\mathrm{LSR}} 2 \mathrm{~km} \mathrm{~s}^{-1}$ to $14 \mathrm{~km} \mathrm{~s}^{-1}$. Contours range between $20 \%$ to $90 \%$ with a step of $10 \%$ of the peak intensities that are $73 \mathrm{~K} \mathrm{~km} \mathrm{~s}$ for ${ }^{12} \mathrm{CO}$ $4-3,72 \mathrm{~K} \mathrm{~km} \mathrm{~s}^{-1}$ for ${ }^{12} \mathrm{CO} 1-0$ and $31 \mathrm{~K} \mathrm{~km} \mathrm{~s}^{-1}$ for ${ }^{13} \mathrm{CO} 1-0$. The seven filled triangles indicates the positions where we carry out a detailed PDR analysis later. The open pentacle symbol denotes the position of HD 281159.

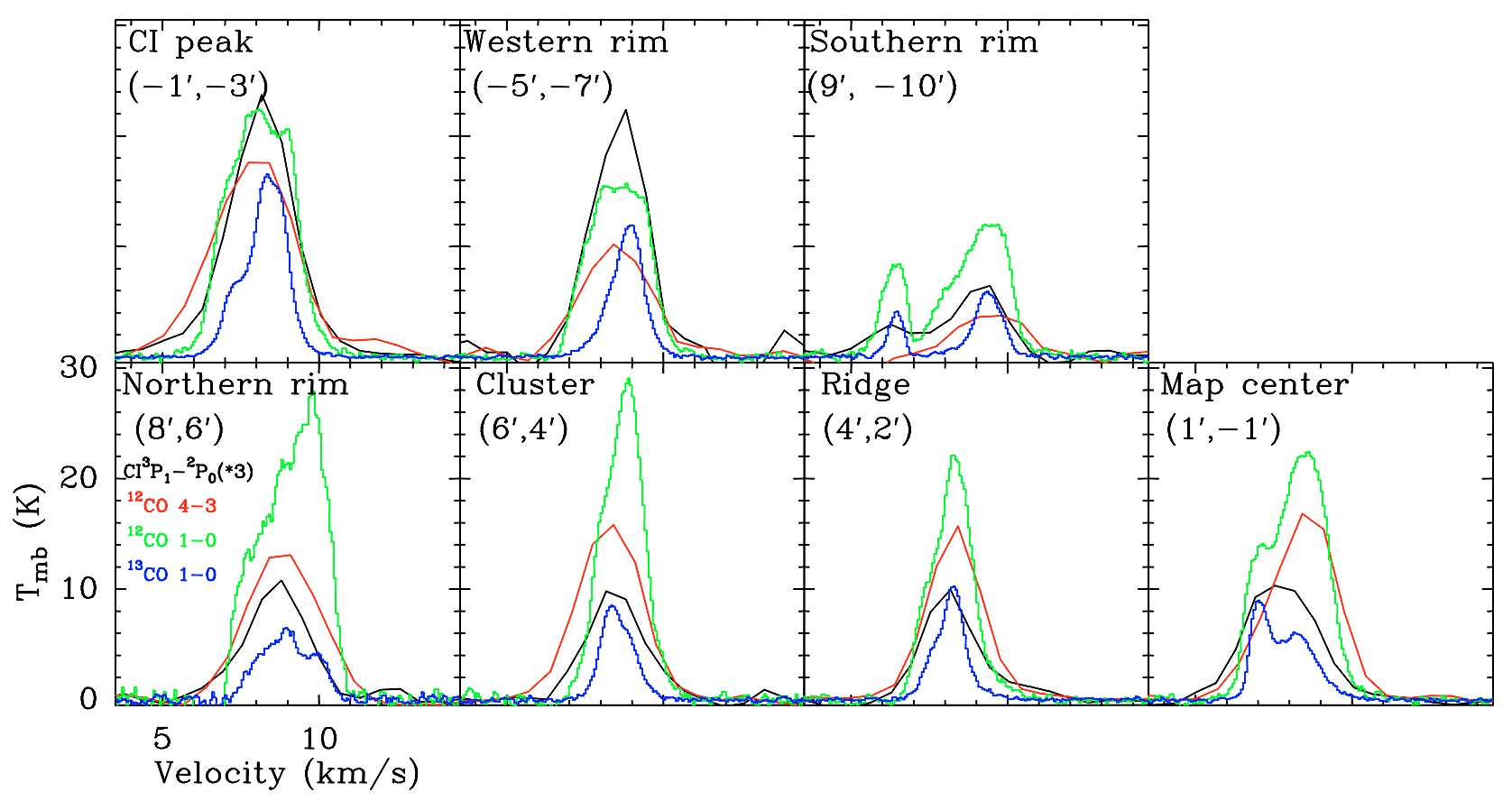

Fig. 2. Spectra of the four different tracers on those six positions along the cut from the northern edge of the cloud into the cloud, and on the seventh position at the south of the cloud. [C I] spectra in all the positions have been multiplied by 3 . All panels have the same $x$ axis from $3.5 \mathrm{~km} \mathrm{~s}^{-1}$ to $14.5 \mathrm{~km} \mathrm{~s}^{-1}$ and the same $y$ axis from $-0.5 \mathrm{~K}$ to $30.5 \mathrm{~K}$.

physical and chemical conditions. Spectra of [CI], ${ }^{12} \mathrm{CO} 4-3$, ${ }^{12} \mathrm{CO} 1-0$ and ${ }^{13} \mathrm{CO} 1-0$ at those seven positions are displayed in Fig. 2.

The $[\mathrm{CI}]$ emission is centered at a velocity of $\sim 8 \mathrm{~km} \mathrm{~s}^{-1}$. The lowest $[\mathrm{CI}]$ main beam brightness temperature $(\sim 2 \mathrm{~K})$ occurs at the southern rim $\left(9^{\prime},-10^{\prime}\right)$, while the highest temperatures of $7.0 \mathrm{~K}$ and $6.7 \mathrm{~K}$ are observed at the position of the [C I] peak $\left(-1^{\prime},-3^{\prime}\right)$ and the western rim $\left(-5^{\prime},-7^{\prime}\right)$, respectively. The line widths of $[\mathrm{CI}]$ spectra at the seven positions range between $2.0 \mathrm{~km} \mathrm{~s}^{-1}$ and $2.6 \mathrm{~km} \mathrm{~s}^{-1}$.
The ${ }^{12} \mathrm{CO} 4-3$ main beam brightness temperature varies between $\sim 3.6 \mathrm{~K}$ at southern rim and $16.4 \mathrm{~K}$ at $[\mathrm{C} \mathrm{I}]$ peak. The line width of the ${ }^{12} \mathrm{CO} 4-3$ spectra ranges between $2.0 \mathrm{~km} \mathrm{~s}^{-1}$ and $2.7 \mathrm{~km} \mathrm{~s}^{-1}$.

Both the ${ }^{12} \mathrm{CO} 1-0$ and ${ }^{13} \mathrm{CO} 1-0$ spectra consist of two velocity components: one is at $\sim 7 \mathrm{~km} \mathrm{~s}^{-1}$ and the other lies at $\sim 8 \mathrm{~km} \mathrm{~s}^{-1}$. The two components are most prominently visible at the southern rim, map center and the $[\mathrm{CI}]$ peak. For the $8 \mathrm{~km} \mathrm{~s}^{-1}$ component at the $[\mathrm{CI}]$ peak, the ${ }^{13} \mathrm{CO} 1-0$ spectrum peaks at the dip of the ${ }^{12} \mathrm{CO} 1-0$ spectrum, which may indicate selfabsorption effect for this component. 
Table 1. The observed integrated line intensities (in $\mathrm{erg} \mathrm{s}^{-1} \mathrm{sr}^{-1} \mathrm{~cm}^{-2}$ ) and line ratios at the seven selected positions. The errors on the integrated intensity are estimated to be $\sim 15 \%$.

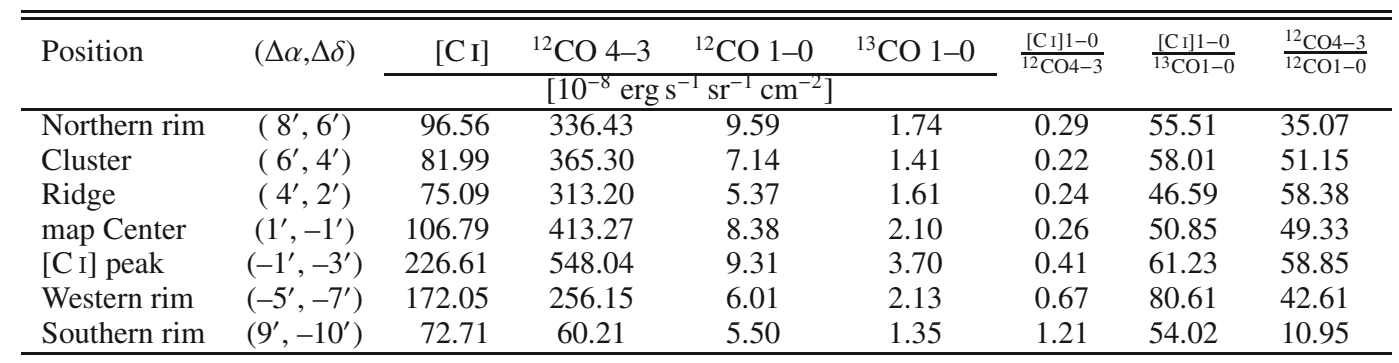
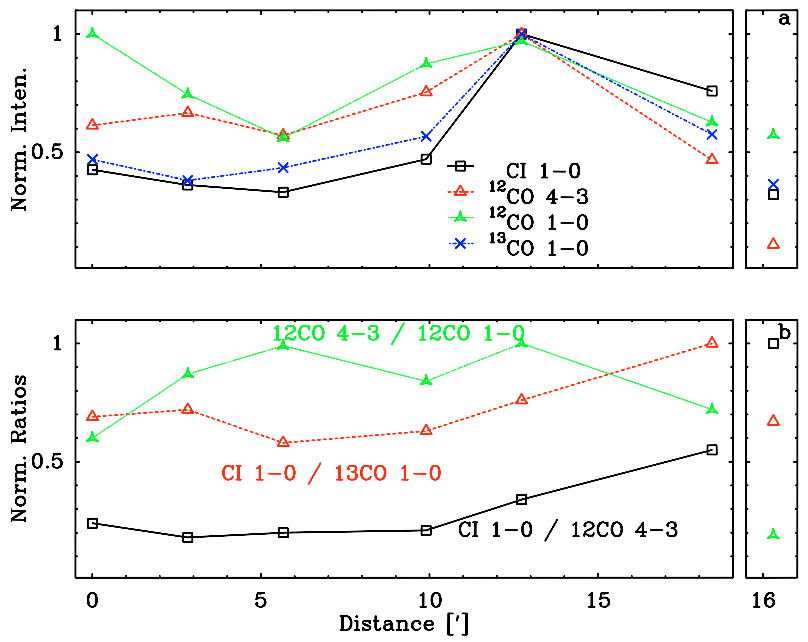

Fig. 3. The top panel a) presents the normalized integrated intensities of $[\mathrm{C} \mathrm{I}],{ }^{12} \mathrm{CO} 4-3,{ }^{12} \mathrm{CO} 1-0$ and ${ }^{13} \mathrm{CO} 1-0$ in the seven selected positions. The typical errors of the intensities are about $15 \%$, resulting in errors of the line ratios of about $21 \%$; b) displays integrated line intensity ratios. The $x$ axes of all panels are the relative distance to the northern rim $\left(8^{\prime}\right.$, $\left.6^{\prime}\right)$ position. The southern rim is plotted in a separate box.

Table 1 presents the integrated intensities and line ratios at the seven selected positions. Figure 3 shows the variation of the integrated intensities and their ratios at these seven positions.

In Fig. $3 \mathrm{a}$ we see that the intensities of $[\mathrm{CI}],{ }^{13} \mathrm{CO} 1-0$ and ${ }^{12} \mathrm{CO} 4-3$ lines show similar trends, only the falling off of the intensity of ${ }^{12} \mathrm{CO} 4-3$ is somewhat less drastic than [C I], ${ }^{13} \mathrm{CO}$ $1-0$. As opposed to the single peak seen in $[\mathrm{C} \mathrm{I}],{ }^{13} \mathrm{CO} 1-0$ and ${ }^{12} \mathrm{CO} 4-3$, the ${ }^{12} \mathrm{CO} 1-0$ intensity profile shows two peaks with comparable intensities, one at the northern rim and the other at the position of the $[\mathrm{CI}$ ] peak.

Three independent line ratios, i.e., [C I] / ${ }^{12} \mathrm{CO} 4-3$, [C I] / ${ }^{13} \mathrm{CO} 1-0$ and ${ }^{12} \mathrm{CO} 4-3 /{ }^{12} \mathrm{CO} 1-0$, are presented in Table 1 and Fig. 3b (in erg s${ }^{-1} \mathrm{sr}^{-1} \mathrm{~cm}^{-2}$ ). The largest $[\mathrm{CI}] /{ }^{12} \mathrm{CO} 4-3$ ratio (1.21) occurs at the southern rim and the second (0.67) and third (0.41) largest are at the western rim and [C I] peak, while the ratios at the other four positions are $\sim 0.25$. When comparing with other star forming regions like W 3 Main, S 106 and Orion A etc. (see Table 3 by Kramer et al. 2004), the $[\mathrm{CI}] /{ }^{12} \mathrm{CO} 4-3$ ratio at the other five positions are within the range found in those star forming regions except for the western and southern rim. The highest ratio at the southern rim is close to the ratio found at the center of M 51 and the nucleus position of NGC 4826 (Israel \& Baas 2002). The ${ }^{12} \mathrm{CO} 4-3 /{ }^{12} \mathrm{CO} 1-0$ ratio peaks at [C I] peak and decreases for the positions further away with increasing distance. The $[\mathrm{CI}] /{ }^{13} \mathrm{CO} 1-0$ ratios are rather constant except for the western rim.

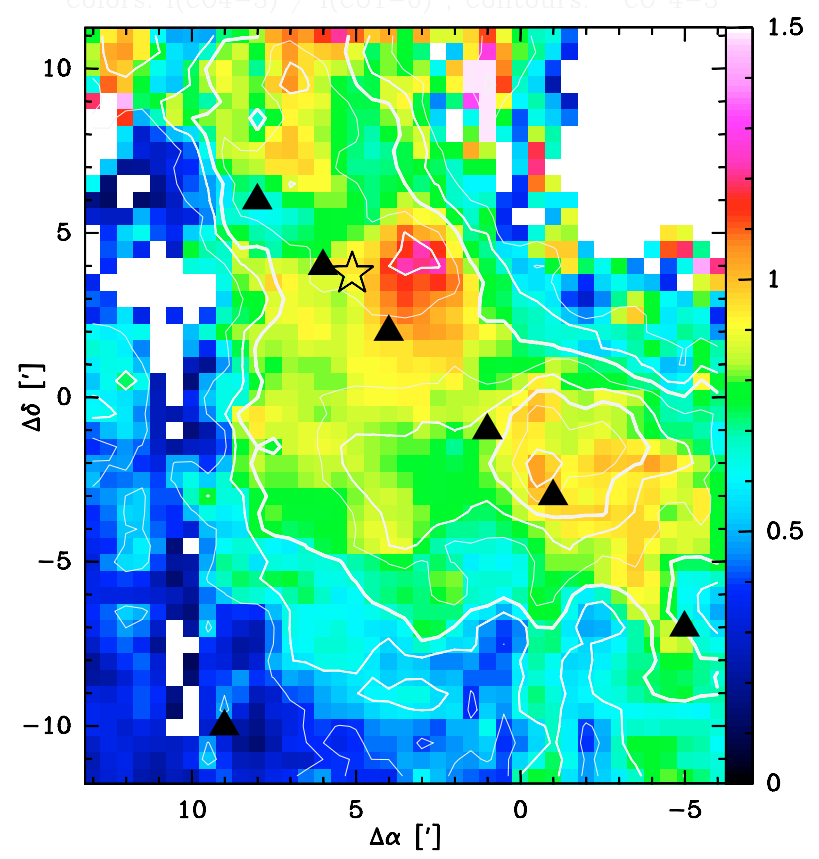

Fig. 4. The color plot represents the ${ }^{12} \mathrm{CO} 4-3 /{ }^{12} \mathrm{CO} 1-0$ line intensity ratio (in units of $\mathrm{K} \mathrm{km} \mathrm{s}^{-1}$ ) observed in the IC 348 cloud. The contours show the ${ }^{12} \mathrm{CO} 4-3$ line intensity spaced at $10 \%$ intervals from $20 \%$ to $90 \%$ relative to the peak intensity of $73 \mathrm{~K} \mathrm{~km} \mathrm{~s}^{-1}$. White pixels in the figure denote positions where either of the line intensities falls below the $3 \sigma$ threshold.

\section{LTE analysis}

Figure 4 shows a map of the ${ }^{12} \mathrm{CO} 4-3 /{ }^{12} \mathrm{CO} 1-0$ line ratio in terms of line integrated temperatures $\left(\mathrm{K} \mathrm{km} \mathrm{s}^{-1}\right)$ overlaid by ${ }^{12} \mathrm{CO} 4-3$ integrated intensities. The ratio has its minimum of $\sim 0.3$ at the edges of the cloud; ratios of $\sim 0.9$ are found at the ${ }^{12} \mathrm{CO} 4-3$ peaks; maximum ratios between 1.1 and 1.5 occur close to HD 281159.

For a rough estimate of the column densities and for an easy comparison to other [C I] studies (e.g. Little et al. 1994; Plume et al. 2000; Bensch et al. 2003) we first apply a Local Thermodynamic Equilibrium analysis. Assuming LTE and optically thick emission, the ${ }^{12} \mathrm{CO} 4-3 /{ }^{12} \mathrm{CO} 1-0$ ratio of 0.3 corresponds to an excitation temperature $T_{\text {ex }}$ of $\sim 10 \mathrm{~K}$. The ratio grows to 0.85 for $T_{\mathrm{ex}} \sim 50 \mathrm{~K}$ and remains almost constant at even higher temperatures. Considering the calibration uncertainty, we set $T_{\mathrm{ex}}$ to $50 \mathrm{~K}$ in all the positions where the ratio falls above 0.85 . We note that $[\mathrm{CI}]$ and ${ }^{12} \mathrm{CO}$ often have different excitation temperatures. However, $N(\mathrm{C})_{\text {LTE }}$ only changes by $20 \%$ when the assumed excitation temperature varies between 20 and 
Table 2. Results of the LTE analysis. The excitation temperature $T_{\mathrm{ex}}$ is listed in Col. (2); Col. (3)-(5) present $\mathrm{C}$, $\mathrm{CO}$ and $\mathrm{H}_{2}$ column densities; the $\mathrm{C} / \mathrm{CO}$ ratio is listed in Col. (6). The uncertainties of the column densities are derived by varying the integrated intensities by $\pm 15 \%$.

\begin{tabular}{|c|c|c|c|c|c|}
\hline Number & $\begin{array}{r}T_{\mathrm{ex}} \\
\mathrm{K}\end{array}$ & $\begin{array}{r}N(\mathrm{C})_{\text {LTE }} \\
10^{16}\left[\mathrm{~cm}^{-2}\right]\end{array}$ & $\begin{array}{c}N(\mathrm{CO})_{\text {LTE }} \\
10^{17}\left[\mathrm{~cm}^{-2}\right]\end{array}$ & $\begin{array}{r}N\left(\mathrm{H}_{2}\right)_{\text {LTE }} \\
10^{21}\left[\mathrm{~cm}^{-2}\right]\end{array}$ & $\mathrm{C} / \mathrm{CO}_{\mathrm{LTE}}$ \\
\hline Northern rim & $18_{15}^{47}$ & $12.08_{9.27}^{19.71}$ & $9.56_{7.16}^{22.84}$ & $12.50_{8.95}^{28.55}$ & $0.13_{0.04}^{0.28}$ \\
\hline Cluster & $50_{20}$ & $9.61_{7.77}^{11.17}$ & $16.99_{7.33}^{19.54}$ & $21.24_{9.16}^{24.43}$ & $0.06_{0.04}^{0.15}$ \\
\hline Ridge & $50_{28}$ & $9.04_{7.32}^{10.46}$ & $19.37_{10.43}^{23.03}$ & $24.21_{13.04}^{28.78}$ & $0.05_{0.03}^{0.10}$ \\
\hline Map center & $48_{18}^{50}$ & $13.09_{10.68}^{16.00}$ & $24.39_{10.25}^{29.03}$ & $\begin{array}{l}30.49_{12.82}^{36.29} \\
\end{array}$ & $0.05_{0.04}^{0.16}$ \\
\hline$\left[\mathrm{C}_{\mathrm{I}}\right]$ peak & $50_{29}^{10}$ & $26.85_{2171}^{30.80}$ & $44.47_{24.56}^{10.23}$ & $55.59^{63.93}$ & $0.06_{0.04}^{0.04}$ \\
\hline Western rim & $28_{15}^{50}$ & $19.72_{17.61}^{28.30}$ & $16.25_{9.52}^{29.99}$ & $20.31_{11.90}^{37.49}$ & $0.12_{0.06}^{0.30}$ \\
\hline Southern rim & $7_{6}^{8}$ & $\begin{array}{l}37.50_{21.96}^{17.57} \\
\end{array}$ & $6.66_{5.40}^{8.54}$ & $8.32_{6.76}^{10.67}$ & $0.56_{0.26}^{1.34}$ \\
\hline
\end{tabular}

$150 \mathrm{~K}$. For the first order estimation, it is therefore reasonable to use the ${ }^{12} \mathrm{CO}$ excitation temperature also for $[\mathrm{C} \mathrm{I}]$. With the ${ }^{12} \mathrm{CO}$ excitation temperatures and the assumption of optically thin $[\mathrm{C} \mathrm{I}]$ and ${ }^{13} \mathrm{CO} 1-0$ emission, we then obtain the column density of $\mathrm{C}$, $N(\mathrm{C})_{\mathrm{LTE}}$, and ${ }^{13} \mathrm{CO}, N\left({ }^{13} \mathrm{CO}\right)_{\mathrm{LTE}}$, from the integrated line intensities. Using the relative abundance ratio $\left[{ }^{12} \mathrm{CO}\right] /\left[{ }^{13} \mathrm{CO}\right]$ of 65 (Langer et al. 1990) we translate the ${ }^{13} \mathrm{CO}$ column densities into $\mathrm{CO}$ column densities. $\mathrm{H}_{2}$ column densities are derived from the $[\mathrm{CO}] /\left[\mathrm{H}_{2}\right]$ abundance ratio of $8 \times 10^{-5}$ (Frerking et al. 1982). The results are summarized in Table 2.

The resulting map of $\mathrm{C} / \mathrm{CO}$ column density ratios is presented in Fig. 5. In most of the cloud, the $\mathrm{C} / \mathrm{CO}$ ratio falls below 0.1 . Higher values (up to 1.5 ) occur at the rim of the cloud where the ${ }^{13} \mathrm{CO} 1-0$ emission is more diffuse. Values as low as 0.02 are found at the southwest of the cloud and along the ridge to the northeast. As ${ }^{13} \mathrm{CO} 1-0$ roughly traces the $\mathrm{H}_{2}$ column density, this plot indicates an anti-correlation between the $\mathrm{C} / \mathrm{CO}$ ratio and the $\mathrm{H}_{2}$ column density. This is consistent with the conclusion of Mookerjea et al. (2006) that the [C I] line is not a tracer of optical extinction, total $\mathrm{H}_{2}$ column density or total mass in the beam.

The seven positions, for which we perform a detailed analysis within the context of a PDR model, exhibit C/CO column density ratios between about 0.05 and 0.5 , i.e. they cover a relatively wide range (see Table 2). The ratios measured within the full map cover almost the full range cited so far in the literature with values as low as 0.03 in parts of G34.3 (Little et al. 1994) and as high as 1.5 seen in the Polaris Flare (Bensch et al. 2003).

\section{PDR modelling}

In the next step we attempt to obtain a self-consistent model for the physical and chemical structure of the cloud matching the observed $\mathrm{C} / \mathrm{CO}$ ratios. We will use a clumpy PDR model to selfconsistently compute the abundance of the species and their excitation determining the line strength. The model is constrained by the strength of the UV field, which can be independently estimated, and by the known geometry, provided by the complex, filamentary, and clumpy structure of the region.

\subsection{FUV intensity}

\subsubsection{Estimate from the FIR continuum}

We use HIRES processed 60 and $100 \mu \mathrm{m}$ IRAS data (Aumann et al. 1990) to generate a far-infrared intensity map $I_{\mathrm{FIR}}$ in IC 348. It represents the far-infrared intensity between $42.5 \mu \mathrm{m}$ and $122.5 \mu \mathrm{m}$ as measured by the filter curves of the two IRAS

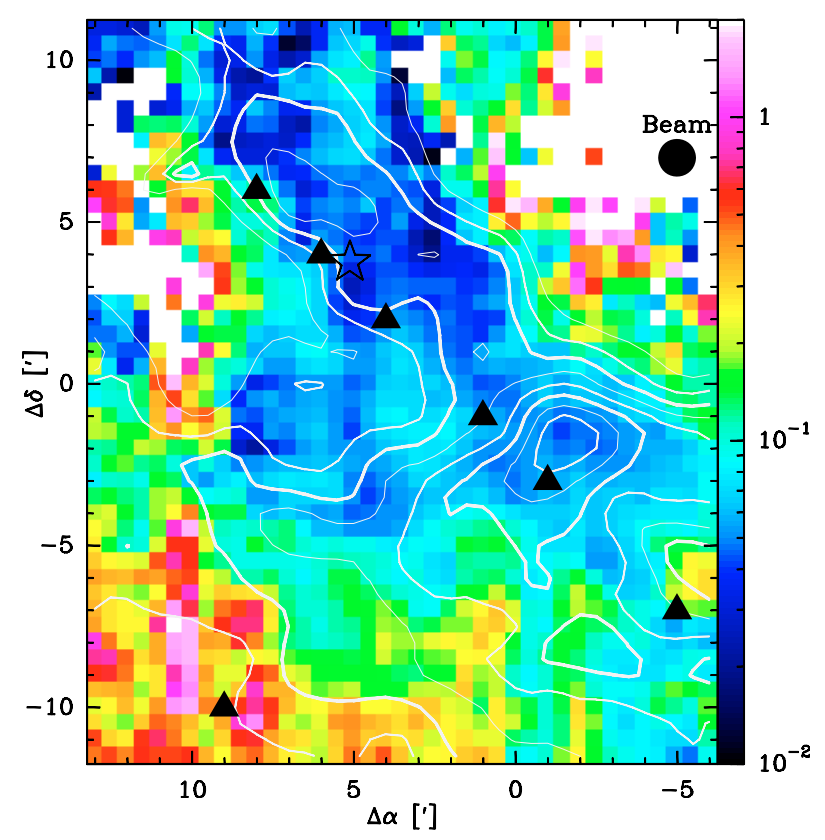

Fig. 5. The $\mathrm{C} / \mathrm{CO}$ column density ratio overlaid by ${ }^{13} \mathrm{CO} 1-0$ integrated intensities contours. The contour levels are spaced at $10 \%$ intervals from $20 \%$ to $90 \%$ relative to the peak intensity of $31 \mathrm{~K} \mathrm{~km} \mathrm{~s}^{-1}$. White pixels in the figure denote positions where the ${ }^{13} \mathrm{CO} 1-0$ intensities fall below the $3 \sigma$ threshold.

data sets (Nakagawa et al. 1998). Following Kaufman et al. (1999), we assume that the total FUV flux, $\chi_{\text {FIR }}$, absorbed by the dust grains is re-radiated in the far-infrared. In this way we estimate the FUV field from the far-infrared field (cf. Kramer et al. 2005): $\chi_{\mathrm{FIR}} / \chi_{0}=4 \pi I_{\mathrm{FIR}}$, where $\chi_{0}=2.7 \times 10^{-3} \mathrm{erg} \mathrm{s}^{-1} \mathrm{~cm}^{-2}$ (Draine 1978; Draine \& Bertoldi 1996). The derived spatial distribution of the FUV intensity is presented in Fig. 6. In the mapped region, the FUV field $\chi_{\text {FIR }}$ varies between about 1 and 100 Draine units. For the seven selected positions $\chi_{\text {FIR }}$ is listed in Table 3.

\subsubsection{Estimate from the stellar radiation}

The primary source of UV radiation in the cloud is HD 281159. Assuming that the star is a black body at an effective temperature $T_{\text {eff }}$ corresponding to its spectral type, we can estimate the FUV flux independently (Kramer et al. 1996; Beuther et al. 2000). A B $5 \mathrm{~V}$ star has an effective temperature $\log \left(T_{\text {eff }}\right)=4.182$ 


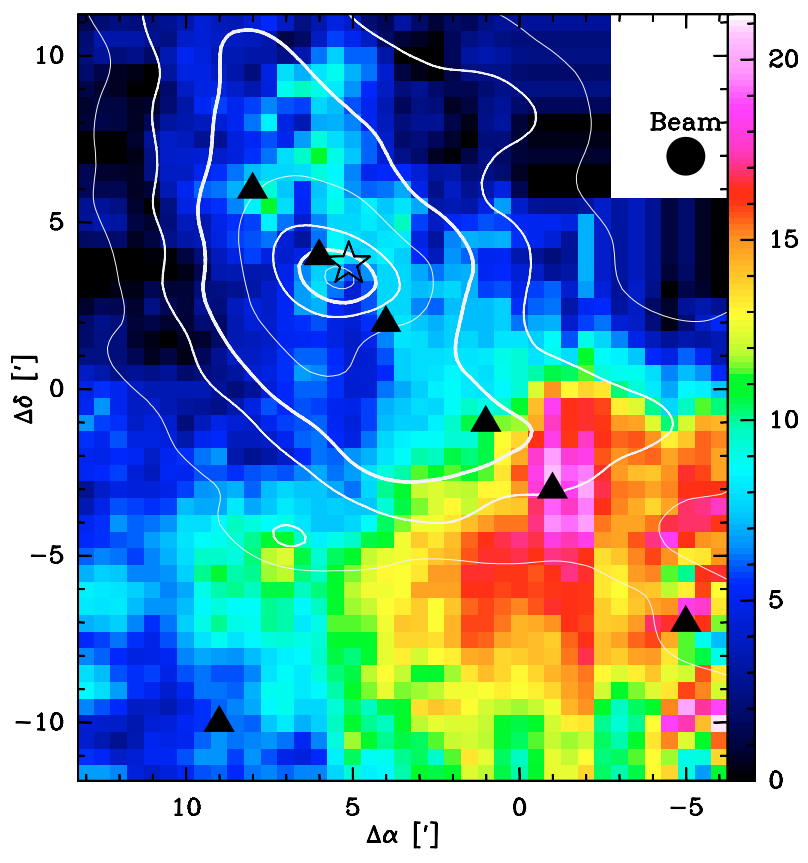

Fig. 6. FUV intensity distribution (contours) in IC 348 estimated from the IRAS FIR fluxes, overlayed on the [C I] integrated intensities (colors). The FUV intensity $\chi_{\text {FIR }}$ contour levels run from 5, 10, 20 to 100 by steps of 20 Draine units, $\chi_{0}$.

and a total luminosity $L$ of $\log \left(L / L_{\odot}\right)=2.681$ (de Jager \& Nieuwenhuijzen 1987). The FUV luminosity $L_{\mathrm{FUV}}$ and the FUV flux $\chi_{\text {FUV }}$ are defined as

$L_{\mathrm{FUV}}=L \cdot \frac{\Phi_{\mathrm{UV}}}{\Phi_{\mathrm{total}}}$, and $\chi_{\mathrm{FUV}}=\frac{L_{\mathrm{FUV}}}{4 \pi \mathrm{d}^{2}}$,

where $\Phi_{\mathrm{UV}}$ is the FUV flux between $6 \mathrm{eV}$ and $13.6 \mathrm{eV}, \Phi_{\text {total }}$ is the total flux, and $d$ is the distance to the UV source. We compute the distance $d$ in two ways: for the point close to HD 281159 we use the optical radius of the cluster IC 348 of about $0.37 \mathrm{pc}$ (Herbig 1998) as the minimum distance between star and cloud. For all other points we assume that star and cloud are located in the same plane so that the distance is directly given by the observed separation. Finally we normalize the FUV field to units of $\chi_{0}$. The resulting FUV fields, $\chi_{\text {star }}$, are also listed in Table 3 for the seven selected positions.

We see that both methods provide consistent FUV field values. Because of the uncertainty of the distance between star and cloud we consider the values $\chi_{\mathrm{FIR}}$, derived from the HIRES data, somewhat more reliable so that we will use them in the following. The FUV field $\chi$ at the seven positions ranges from about 1 to 90 Draine units.

\subsection{Clumpy PDR scenarios}

Molecular clouds are known for their highly filamentary and clumpy, often self-similar structure over a wide range of scales (Dame et al. 2001; Heyer et al. 1998; Stutzki et al. 1988). The observed structure further breaks up into substructures with every step of increased spatial resolution (Falgarone \& Phillips 1996; Bensch et al. 2001). For IC 348, the self-similar structure was directly measured in terms of the $\Delta$-variance by Sun et al. (2006). In such a cloud, the UV field can penetrate deeply into the cloud forming PDRs at many internal surfaces. The cloud is basically filled by surfaces (Ossenkopf et al. 2007). This explains the observed $[\mathrm{CI}]$ and even $[\mathrm{C}$ II] emission deep within molecular clouds (Stutzki et al. 1988; Howe et al. 1991; Stacey et al. 1993; Meixner \& Tielens 1993). We can simulate such a structure by an ensemble of individual clumps, each of them being small compared to the overall cloud, leaving enough "empty" regions to permit a deep penetration of the UV field (Stutzki et al. 1998).

In the following we test two different clump ensembles with respect to their ability to reproduce the observed line intensities.

\subsubsection{Modelling of individual clumps}

All individual clumps in the PDRs are modelled using the spherical KOSMA $-\tau$ PDR model (Störzer et al. 1996; Röllig et al. 2006, 2007). The model computes the chemical and temperature structure of a clump illuminated by an isotropic FUV radiation field and cosmic rays. The emission from the model is calculated as a function of the gas density, FUV radiation field and mass of the clumps (implicitly specifying the clump size). The model clumps are assumed to have a power-law density profile of $n(\mathrm{r})$ $\propto n_{0} r^{-1.5}$ for $0.2 \leq r / r_{\mathrm{cl}} \leq 1$ and $n(\mathrm{r})=$ const. for $r / r_{\mathrm{cl}} \leq 0.2 . n_{0}$ is the density at the clump surface called clump density, which is about half of the clump averaged density $\langle n\rangle: n_{0} \sim \frac{1}{1.91}\langle n\rangle$.

The PDR models are pre-computed on a regular grid with equidistant logarithmic steps. The FUV field $\chi$ covers the range from $10^{0}, 10^{0.5}, \ldots, 10^{5.5}, 10^{6.0}$ Draine units; The clump densities range from $10^{2.0}, 10^{2.5}, \ldots, 10^{5.5}, 10^{6.0} \mathrm{~cm}^{-3}$; and the clump masses $M_{\mathrm{cl}}$ cover $10^{-3.0}, 10^{-2.5}, \ldots, 10^{1.5}, 10^{2.0} M_{\odot}$.

\subsubsection{Ensemble of identical clumps (Ensemble 1)}

In a first approach we will assume that the cloud is composed of a collection of identical clumps, so that the full ensemble is characterized by the parameter set of the individual clumps and their number only. To determine the properties of the individual clumps we perform a $\chi^{2}$ fit of the three line intensity ratios given in Table 1, using a spherical PDR model in a three-dimensional parameter space spanned by the FUV field, the clump mass, and density. The results for the seven selected positions are summarized in Table 3. Figure 7 presents an example of the $\chi^{2}$ fitting for the map center $\left(1^{\prime},-1^{\prime}\right)$. The left panel shows a cut through the parameter space for a fixed UV field, demonstrating the fit of clump mass $M_{\mathrm{cl}}$ and clump density $n_{0}$. The right panel presents the corresponding fit for the FUV field $\chi$ and the clump density $n_{0}$. We see that all line ratios are good density tracers, but hardly constrain the clump mass as expected. The clump density has to fall between 2.5 and $5.0 \times 10^{5} \mathrm{~cm}^{-3}$. But the reduced $\chi^{2}$ only changes between 1 and 2.5 when the clump mass varies from $10^{-2.0}$ to $10^{1.0} M_{\odot}$. This general behaviour applies to all positions analyzed. The clump densities at the seven positions vary between $4.4 \times 10^{4}$ to $4.3 \times 10^{5} \mathrm{~cm}^{-3}$. The highest clump density occurs at positions of the ridge and the map center. The clump masses are not well constrained, the best fitting values fall between 0.1 and $0.4 M_{\odot}$. The fitted FUV flux $\chi_{\text {PDR }}$ agrees with $\chi_{\text {FIR }}$ derived from the FIR continuum in most positions. The maximum deviations by a factor three correspond to the maximum deviation that we got comparing the FIR continuum with the stellar radiation estimate.

The total number of clumps in the beam $N_{\text {ens }}$ is calculated by comparing the observed absolute line integrated intensities with the models, correcting for the beam filling factor ${ }^{2}$. A $70^{\prime \prime}$ beam

\footnotetext{
2 By using line integrated intensities, a smaller internal velocity distribution in the clumps relative to the total observed line width will be automatically corrected by a higher clump number.
} 

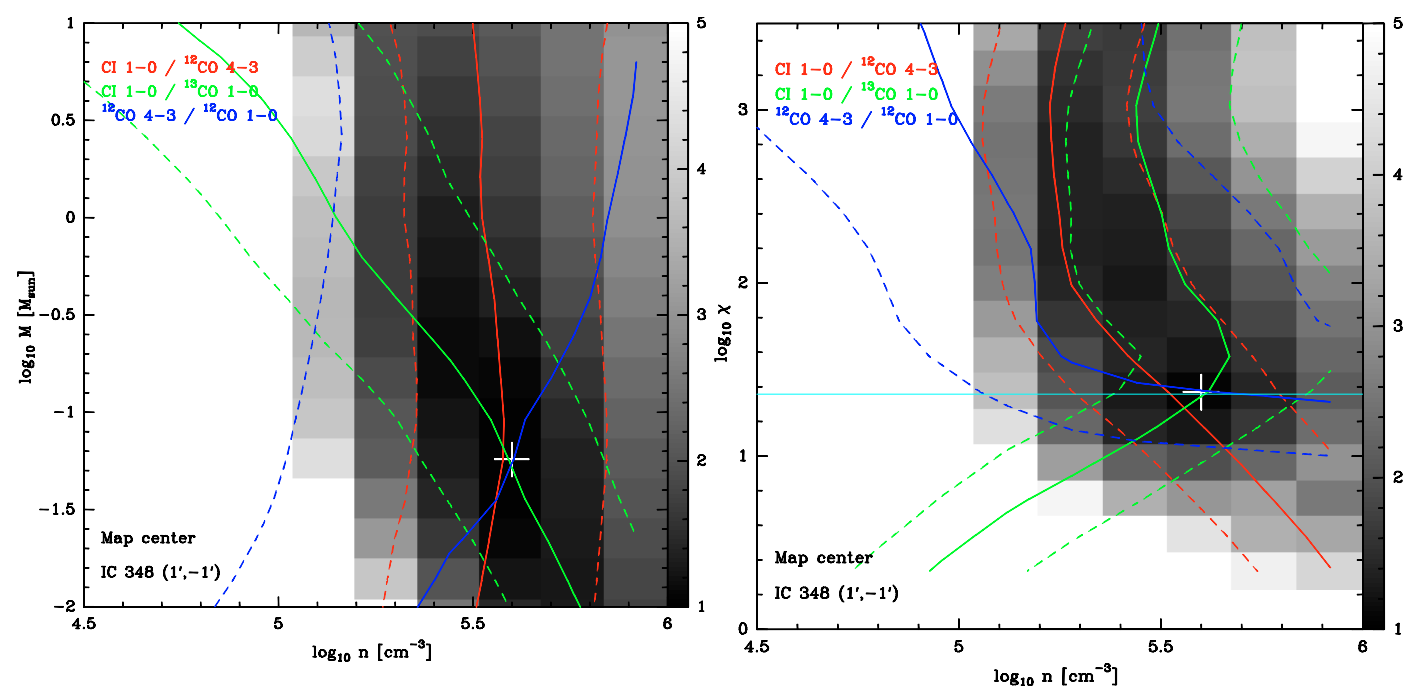

Fig. 7. Comparison of the observed line intensity ratios $[\mathrm{C} \mathrm{I}] /{ }^{12} \mathrm{CO} 4-3,[\mathrm{CI}] / /^{13} \mathrm{CO} 1-0$ and ${ }^{12} \mathrm{CO} 4-3 /{ }^{12} \mathrm{CO} 1-0$ at $\left(1^{\prime},-1^{\prime}\right)$ with the single clump KOSMA - $\tau$ PDR model calculations. The left figure shows the fit of clump mass and density at a fixed FUV field $\chi$ of $10^{1.5}$; the right one presents the fit of FUV field and clump density at a fixed clump mass of $10^{-1} M_{\odot}$. The central solid contours represent the observed intensity ratios, the outer dashed two give those for a $20 \%$ uncertainty. The grey-scale images indicate the reduced $\chi^{2}$ of the fit. The position of the minimum reduced $\chi^{2}$ is marked by a white cross. The horizontal light blue line in the right panel shows the FUV flux $\chi_{\text {FIR }}$ from the HIRES data.

Table 3. Physical parameters at selected positions in Fig 1, obtained by fitting the observed intensity ratios to the single clump KOSMA $-\tau$ PDR models. Cols. (2)-(5) list the fitted clump densities, clump mass, clump radius and FUV field; Col. (6) is the minimum reduced $\chi^{2}$ of the PDR fit; the FUV field derived from the FIR continuum $\chi_{\text {FIR }}$ and that from the stellar radiation $\chi_{\text {star }}$ are in Cols. (7) and (8); The distance to HD 281159 used for the calculation of $\chi_{\text {star }}$ is listed in Col. (9).

\begin{tabular}{lcccccccc}
\hline \hline Position & $\begin{array}{c}n \\
{\left[10^{5} \mathrm{~cm}^{-3}\right]}\end{array}$ & $\begin{array}{c}M_{\mathrm{cl}} \\
M_{\odot}\end{array}$ & $\begin{array}{c}R_{\mathrm{cl}} \\
\mathrm{pc}\end{array}$ & $\chi_{\mathrm{PDR}}$ & $\chi_{\text {min }}^{2}$ & $\chi_{\mathrm{FIR}}$ & $\chi_{\mathrm{star}}$ & $\begin{array}{c}d \\
\mathrm{pc}\end{array}$ \\
\hline Northern rim & 1.91 & 0.2 & 0.01 & 15 & 1.38 & 39 & 40 & 0.37 \\
Cluster & 2.75 & 0.1 & 0.01 & 98 & 1.05 & 84 & 40 & 0.37 \\
Ridge & 4.25 & 0.4 & 0.01 & 38 & 1.23 & 46 & 40 & 0.37 \\
Map center & 3.98 & 0.1 & 0.01 & 23 & 1.04 & 23 & 13 & 0.64 \\
[C I] peak & 1.91 & 0.1 & 0.02 & 23 & 1.96 & 11 & 7 & 0.90 \\
Western rim & 0.63 & 0.2 & 0.02 & 20 & 2.00 & 6 & 3 & 1.42 \\
Southern rim & 0.44 & 0.4 & 0.02 & 2 & 2.31 & 1 & 3 & 1.29 \\
\hline
\end{tabular}

corresponds to a diameter of $0.10 \mathrm{pc}$ at the distance of IC 348 . The clump size obtained from the best fit models found in the previous section ranges from 0.01 to $0.02 \mathrm{pc}$. The beam filling factor $\eta_{\mathrm{bf}, \mathrm{i}}$ of each clump is calculated as $\eta_{\mathrm{bf}, \mathrm{i}}=\Omega_{\mathrm{cl}} / \Omega_{\text {beam }}$, where $\Omega_{\mathrm{cl}}$ and $\Omega_{\text {beam }}$ are the solid angles of a clump and the beam. $\Omega_{\mathrm{cl}}=\pi R_{\mathrm{cl}}^{2} / D^{2}$ is the solid angle of the individual clump. $D$ is the distance of the cloud; assuming a Gaussian beam shape, $\Omega_{\text {beam }}$ is computed as $\Omega_{\text {beam }}=\pi \theta_{\text {beam }}^{2} / 4 \ln 2$, where $\theta_{\text {beam }}$ is the FWHM of our beam. The intensity correction for a single clump is $I_{\mathrm{i}}^{\prime}=I_{\mathrm{mb}, \mathrm{i}} / \eta_{\mathrm{bf}, \mathrm{i}}$, where $I_{\mathrm{mb}, \mathrm{i}}$ is the beam averaged line intensity for that single clump. Since $[\mathrm{CI}]$ is the most optically thin one among our four tracers, we use its line intensity to derive the number of clumps in the beam $N_{\text {ens }}$ (see Table 4) by dividing the observed intensities $\left.I_{[\mathrm{C}}\right]$ obs by the intensities for a single clump $I_{[\mathrm{C} \text { I }] \bmod , \mathrm{i}}$

$N_{\mathrm{ens}}=\frac{I_{[\mathrm{C} \mathrm{I}] \mathrm{obs}}}{I_{[\mathrm{C} \mathrm{I}] \mathrm{mod}, \mathrm{i}} \cdot \eta_{\mathrm{bf}, \mathrm{i}}}=\frac{I_{[\mathrm{C} \mathrm{I}] \mathrm{obs}}}{I_{[\mathrm{C} \mathrm{I}] \mathrm{mod}, \mathrm{i}}} \times \frac{\Omega_{\mathrm{beam}}}{\Omega_{\mathrm{cl}}}$.

Then we use $N_{\text {ens }}$ to calculate the ratios $R$ of line intensities between model ensemble and observations for the other tracers:

$R=\frac{N_{\mathrm{ens}} \cdot I_{\mathrm{mod}, \mathrm{i}} \cdot \eta_{\mathrm{bf}, \mathrm{i}}}{I_{\mathrm{obs}}}$

The $R$ values are listed in Table 4. This allows easy judgment of the quality of the model fit. Considering the calibration uncertainty, a perfectly fitting model yields a ratio $R$ of $1 \pm 0.15$. All three remaining tracers from Ensemble 1 have a good agreement between the modelled and observed absolute line intensities.

\subsubsection{Clumps with a mass and size spectrum (Ensemble 2)}

Representing the clumpy structure of the cloud by an ensemble of identical clumps is, of course, an oversimplification. A realistic clump size and mass distribution should be taken into account. Large-scale CO maps present the clumpy structure of the ISM with a mass distribution following a power law $\mathrm{d} N / \mathrm{d} M \propto M^{-\alpha}$, where $N$ is the number of clumps and $\alpha$ has a value around 1.8 (Kramer et al. 1998b). Furthermore, the observations also show that there is a strong correlation of the density and mass of the clumps corresponding a mass-size relation $r \propto M^{\gamma}$ with $\gamma \approx 2.3$ (Heithausen et al. 1998). For an ensemble of randomly positioned clumps with a power law mass spectrum, there is a relation among the power law spectral index $\beta$ of the power spectrum, $\alpha$ and $\gamma: \beta=\gamma(3-\alpha)$ (Stutzki et al. 1998). Using the $\alpha$ and $\gamma$ values above, $\beta=2.76$, which is close to the power law index $\beta=2.71$ of ${ }^{13} \mathrm{CO} 2-1$ in IC 348 found by Sun et al. (2006).

In this section we assume these characteristics to be universal and model the emission by ensemble averaging the PDR single clump results over such a clump distribution (Cubick 2005; Cubick et al. 2008) to reproduce the observed line intensities. 
Table 4. Results of ensemble of identical clumps, Ensemble case 1. The mass and FUV field for each single clump are listed in Cols. (2) and (3); Cols. (4)-(6) present the line intensity ratios between the ensemble models and observations of ${ }^{12} \mathrm{CO} 4-3,{ }^{12} \mathrm{CO} 1-0$ and ${ }^{13} \mathrm{CO} 1-0$; the $\mathrm{C}$, CO and $\mathrm{H}_{2}$ column densities from the ensembles are presented in Cols. (7)-(9); Col. (10) is the C/CO ratios. The total number of the clumps in the ensemble and ensemble mass are listed in Cols. (11) and (12); Col. (13) lists the beam filling factor of each clump. The total ensemble mass $M_{\text {ens }}$ listed in the table is computed as $M_{\mathrm{ens}}=N_{\mathrm{ens}} \times M_{\mathrm{cl}}$, where $M_{\mathrm{cl}}$ is the mass of a clump.

\begin{tabular}{|c|c|c|c|c|c|c|c|c|c|c|c|c|}
\hline Position & $\begin{array}{l}M_{\mathrm{cl}} \\
M_{\odot}\end{array}$ & $\chi_{\mathrm{en} 1}$ & $R_{12} \mathrm{CO} 4-3$ & $R_{12} \mathrm{CO}-0$ & $R_{13} \mathrm{CO} 1-0$ & $\begin{array}{r}N(\mathrm{C})_{\text {ens }} \\
10^{16}\left[\mathrm{~cm}^{-2}\right]\end{array}$ & $\begin{array}{r}N(\mathrm{CO})_{\text {ens }} \\
10^{17}\left[\mathrm{~cm}^{-2}\right]\end{array}$ & $\begin{array}{r}N\left(\mathrm{H}_{2}\right)_{\mathrm{ens}} \\
10^{21}\left[\mathrm{~cm}^{-2}\right]\end{array}$ & $\mathrm{C} / \mathrm{CO}_{\mathrm{ens}}$ & $N_{\text {ens }}$ & $\begin{array}{c}M_{\text {ens }} \\
M_{\odot}\end{array}$ & $\eta_{\mathrm{bf}, \mathrm{i}}$ \\
\hline Northern rim & 0.32 & 10 & 0.63 & 0.67 & 0.87 & 13.97 & 18.58 & 7.72 & 0.075 & 4.59 & 1.47 & 0.17 \\
\hline Cluster & 0.10 & 100 & 1.07 & 0.99 & 1.13 & 10.02 & 21.56 & 8.90 & 0.046 & 16.75 & 1.68 & 0.04 \\
\hline Ridge & 0.32 & 32 & 0.95 & 1.13 & 0.95 & 8.90 & 29.49 & 11.29 & 0.030 & 6.72 & 2.15 & 0.08 \\
\hline Map center & 0.10 & 32 & 0.94 & 1.05 & 0.97 & 13.25 & 34.24 & 13.05 & 0.039 & 24.56 & 2.46 & 0.04 \\
\hline$[\mathrm{C}$ I] peak & 0.10 & 32 & 0.94 & 1.59 & 0.83 & 31.54 & 29.88 & 13.14 & 0.106 & 24.74 & 2.47 & 0.08 \\
\hline Western rim & 0.10 & 10 & 0.78 & 1.43 & 0.71 & 24.52 & 9.33 & 6.10 & 0.263 & 11.51 & 1.15 & 0.17 \\
\hline Southern rim & 0.32 & 1 & 0.84 & 0.81 & 0.74 & 14.62 & 6.28 & 3.03 & 0.233 & 5.70 & 1.82 & 0.17 \\
\hline
\end{tabular}

Table 5. Results of ensembles with a clump mass distribution and mass-size distribution, Ensemble case 2. Column (2)-(4) present the ratios (in erg s${ }^{-1} \mathrm{sr}^{-1} \mathrm{~cm}^{-2}$ ) of the modelled and observed line intensities for ${ }^{12} \mathrm{CO} 4-3,{ }^{12} \mathrm{CO} 1-0$ and ${ }^{13} \mathrm{CO} 1-0$; The mean clump ensemble density $n_{\text {mean }}$ and the fitted FUV field $\chi_{\text {en2 }}$ are presented in Cols. (5) and (6). The C, CO and $\mathrm{H}_{2}$ column densities from the ensembles are presented in Cols. (7)-(9); Col. (10) is the C/CO ratios. The total masses are listed in Col. (11); Col. (12) is the minimum reduced $\chi^{2}$ of the fit.

\begin{tabular}{|c|c|c|c|c|c|c|c|c|c|c|c|}
\hline Position & $R_{12} \mathrm{CO} 4-3$ & $R_{12} \mathrm{CO}-0$ & $R_{13} \mathrm{CO}-0$ & $\begin{array}{c}n_{\text {mean }} \\
{\left[10^{5} \mathrm{~cm}^{-3}\right]}\end{array}$ & $\chi_{\mathrm{en} 2}$ & $\begin{array}{r}N(\mathrm{C})_{\mathrm{ens}} \\
10^{16}\left[\mathrm{~cm}^{-2}\right]\end{array}$ & $\begin{array}{r}N(\mathrm{CO})_{\text {ens }} \\
10^{17}\left[\mathrm{~cm}^{-2}\right]\end{array}$ & $\begin{array}{r}N\left(\mathrm{H}_{2}\right)_{\text {ens }} \\
10^{21}\left[\mathrm{~cm}^{-2}\right]\end{array}$ & $\mathrm{C} / \mathrm{CO}_{\mathrm{ens}}$ & $\begin{array}{c}M_{\text {ens }} \\
M_{\odot}\end{array}$ & $\chi_{\min }^{2}$ \\
\hline Northern rim & 1.00 & 1.04 & 1.44 & 3.2 & 3 & 14.36 & 51.82 & 19.28 & 0.028 & 3.84 & 1.43 \\
\hline Cluster & 1.06 & 1.03 & 1.13 & 3.2 & 100 & 9.88 & 25.58 & 9.93 & 0.039 & 1.97 & 0.23 \\
\hline Ridge & 1.13 & 1.26 & 0.91 & 3.2 & 32 & 9.05 & 23.43 & 9.10 & 0.039 & 1.83 & 0.50 \\
\hline Map center & 1.08 & 1.19 & 1.08 & 3.2 & 10 & 13.78 & 40.71 & 15.39 & 0.034 & 3.07 & 0.29 \\
\hline [C I] peak & 1.13 & 1.73 & 0.86 & 1.0 & 10 & 31.24 & 40.16 & 16.61 & 0.078 & 2.88 & 2.37 \\
\hline Western rim & 1.83 & 2.03 & 1.13 & 1.0 & 10 & 30.49 & 23.72 & 12.61 & 0.078 & 2.19 & 3.37 \\
\hline Southern rim & 1.06 & 0.84 & 0.66 & 0.3 & 1 & 13.58 & 5.24 & 2.62 & 0.259 & 0.53 & 4.91 \\
\hline
\end{tabular}

The remaining parameter space is then given by the FUV field input $\chi_{\text {ens }}$, the total mass within the beam $M_{\text {ens }}$, the average clump ensemble density $n_{\text {mean }}$, and the minimum and maximum clump masses, $m_{\mathrm{cl}}^{\min }$ and $m_{\mathrm{cl}}^{\max }$.

To constrain the fit to a three-dimensional parameter space also for this case, we fix the clump mass limits. The upper mass limit has to reflect the total mass of the clump distribution because a spectral index $\alpha=1.8$ corresponds to a top-heavy function, i.e. a distribution where most of the mass is contained in the few most massive clumps. We assume that the total mass obtained from the Ensemble 1 model also provides a reasonable guess for the total mass in Ensemble 2, consequently setting the upper mass limit $m_{\mathrm{cl}}^{\max }$ to half of the total mass of Ensemble 1 . We set the lower mass limit, $m_{\mathrm{cl}}^{\min }$, to $10^{-2} M_{\odot}$. These small clumps can only exist transiently and evaporate on a time scale of $\sim 5000 \mathrm{yrs}$ (Kramer et al. 2008). The choice of the lower mass limit is not critical for the fit. Cubick et al. (2008) studied the dependencies of the model output on the clump mass limits and found that mainly high $-J$ CO transitions (like ${ }^{12} \mathrm{CO} 8-7$ ) are sensitive to the lower clump mass limit.

The actual fit is performed analogously to the ensemble 1 case. The results are presented in Table 5 . We find a reasonable agreement between the modelled line intensities from Ensemble 2 and the observed ones. The intensity ratios between model and observations range between 0.7 and 2.0. The fitted FUV field $\chi_{\text {en } 2}$ from Ensemble 2 is consistent with the field $\chi_{\text {ens }}$ from Ensemble 1 and that derived from the FIR continuum in most positions. The maximum deviation occurs at the northern rim.

The reduced $\chi^{2}$ values are similar for both ensembles at values less than 5. In spite of the somewhat more complex model, the fit is not better than for an ensemble of identical clumps (Ensemble 1).
To compare the observed absolute line intensities with the model prediction, we show two examples of observed and modelled integrated intensities versus frequency, namely the cooling curves of ${ }^{12} \mathrm{CO},{ }^{13} \mathrm{CO}$ (upto $J=9-8$ ) and [C I] for the best-fit of both Ensemble 1 and Ensemble 2 models at the northern rim and the southern rim (see Fig. 8). At those two positions the modelled and observed line intensities agree very well. Figure 8 also provides a prediction for line intensities of $[\mathrm{C} \mathrm{I}]{ }^{3} \mathrm{P}_{2}-{ }^{3} \mathrm{P}_{1},{ }^{12} \mathrm{CO}$ $7-6,{ }^{13} \mathrm{CO} 8-7$, etc. Figure 8 indicates that the tracers studied in this paper do not allow to draw any conclusion about the clump size spectrum that best fits the observations. Both Ensemble 1 and 2 fit the observed data equally well. On the other hand, they give very different predictions for the higher $J$ CO lines. The line intensities predicted for Ensemble 2 are noticeably larger than those predicted for Ensemble 1 (see Fig. 8). The ${ }^{13} \mathrm{CO} 8-7$ line, tracing the column densities of the warm gas, is predicted to be very sensitive to the differences between the two models, allowing to discriminate between them.

Based on the $\mathrm{CO}$ and $\mathrm{H}_{2}$ column densities from the PDR analysis, we find the averaged $\mathrm{CO}$ relative abundance $[\mathrm{CO}] /\left[\mathrm{H}_{2}\right]$ is $\sim 2.4 \times 10^{-4}$, which is 3 times larger than the canonical ratio $[\mathrm{CO}] /\left[\mathrm{H}_{2}\right]$ of $8 \times 10^{-5}$ (Frerking et al. 1982) that we used in the LTE analysis. The beam averaged $\mathrm{C} / \mathrm{CO}$ abundance ratio (Table 5) varies by a factor 10 between 0.03 at the northern rim, which shows the highest column density of all studied positions of $2 \times 10^{21} \mathrm{~cm}^{-2}$, and 0.26 at the southern rim, which shows the lowest $\mathrm{H}_{2}$ column density of $3 \times 10^{21} \mathrm{~cm}^{-2}$. The observed anti-correlation between $\mathrm{C} / \mathrm{CO}$ and $N\left(\mathrm{H}_{2}\right)$ and also the absolute values resemble those found in many other Galactic molecular clouds as compiled recently by Mookerjea et al. (2006). They used the KOSMA- $\tau$ model to interpret data taken in the Cepheus B star forming region. They suggested that the emission of high column density peaks is dominated by massive clumps 

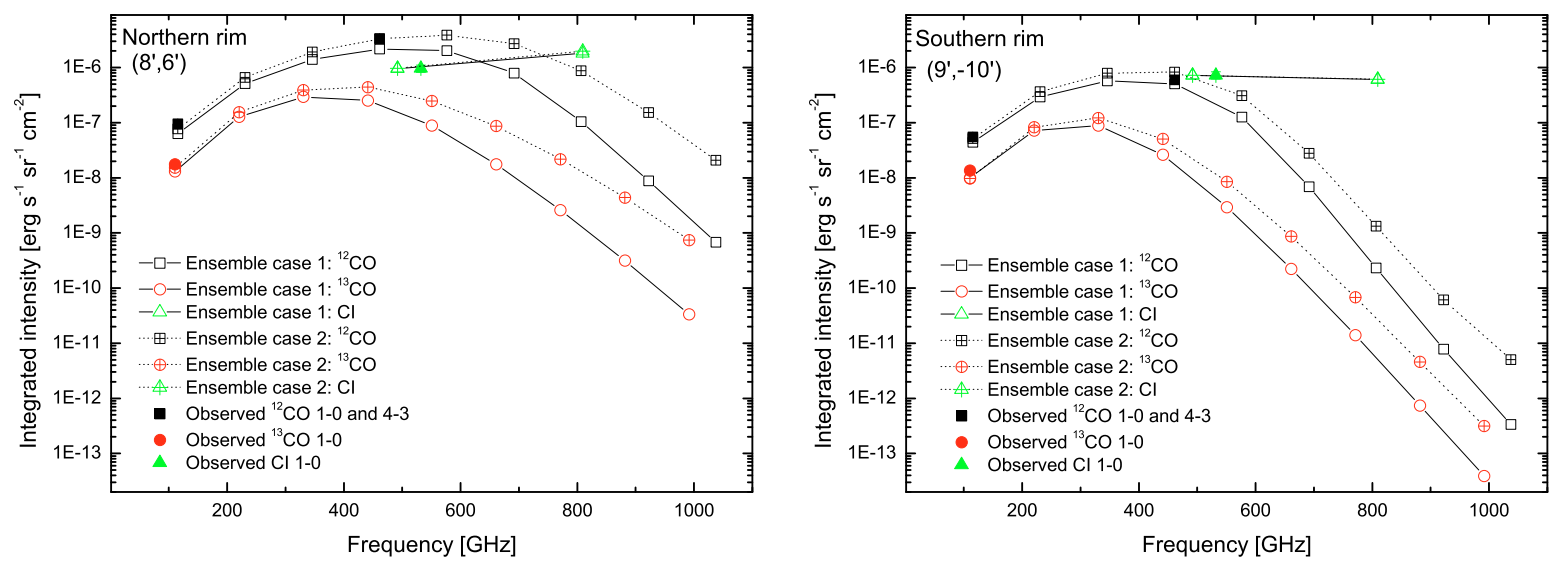

Fig. 8. Observed (filled symbols) and modelled line intensities of ${ }^{12} \mathrm{CO},{ }^{13} \mathrm{CO}$, and $[\mathrm{C} \mathrm{I}]$ from both Ensemble case 1 (drawn lines with open symbols) and Ensemble case 2 (dotted lines, open symbols with a cross inside) at the northern rim (left panel) and the southern rim (right panel). To better display the data, we artificially shift the [C I] frequency by $+40 \mathrm{GHz}$ for the observed intensity.

exhibiting low $\mathrm{C} / \mathrm{CO}$ ratios, while positions of low column densities are dominated by smaller clumps, exhibiting higher $\mathrm{C} / \mathrm{CO}$ ratios. This scenario is confirmed in IC 348 by a more complete analysis using clump mass ensembles following the canonical mass and size distributions. However, the scatter of the present data is large. Observations of the higher lying transitions of [C I] and $\mathrm{CO}$ are needed to further improve the analysis.

\section{Summary and conclusions}

We have presented fully sampled [CI] and ${ }^{12} \mathrm{CO} 4-3$ maps of the IC 348 molecular cloud, covering a region of $20^{\prime} \times 20^{\prime}$. The observed ${ }^{12} \mathrm{CO} 4-3 /{ }^{12} \mathrm{CO} 1-0$ ratios vary between 0.2 and 1.5 . High ${ }^{12} \mathrm{CO} 4-3 /{ }^{12} \mathrm{CO} 1-0$ ratios occur near the B 5 star, at the cloud center and northern edge of the cloud.

We have estimated the FUV field from the FIR continuum intensities obtained from the 60 and $100 \mu \mathrm{m}$ HIRES/IRAS images. The FUV field in the whole observed region ranges between 1 and 100 Draine units. We also used HD 281159, the primary source of UV radiation in this region, to estimate the FUV field for the seven studied regions and the FUV field varies between 3 to 40 Draine units.

Using a clumpy PDR model based on the spherical KOSMA $-\tau$ PDR model we fitted the observed line intensities and intensity ratios at seven selected positions. The observed intensity ratios provide a strong constraint on the clump densities. They fall between $4.4 \times 10^{4} \mathrm{~cm}^{-3}$ and $4.3 \times 10^{5} \mathrm{~cm}^{-3}$. The FUV field fitted in the model falls between 2 to 100 Draine units, consistent with independent estimates for the FUV field derived from the FIR continuum maps by IRAS and from the stellar radiation. We found that both an ensemble of identical clumps (Ensemble 1) and a model using a clump size and mass distribution following the typically observed scaling (Ensemble 2) produce line intensities which are in good agreement (within a factor $\sim 2$ ) with the observed intensities. The models confirm the anti-correlation between $\mathrm{C} / \mathrm{CO}$ abundance ratio and hydrogen column density found in many regions and explained by Mookerjea et al. (2006).

The results of this study strengthen the picture of molecular clouds as highly fragmented, surface dominated objects. Although Ensemble 2 is the natural case in this picture, Ensemble 1 fits the observed intensities equally well. We predicted line intensities for $[\mathrm{CI}], \mathrm{CO}$, and ${ }^{13} \mathrm{CO}$ transitions for both cases, which can be tested by future observations. The higher $J$ CO lines are needed to decide on the necessity of the clump mass and size distributions and to determine the clump mass limits (Cubick et al. 2008).

Acknowledgements. We thank Naomi Ridge for allowing us to use the FCRAO low- $J$ CO data in IC 348. We used the NASA/IPAC/IRAS/HIRES data reduction facilities. This material is based on work supported by the Deutsche Forschungs Gemeinschaft (DFG) via grant SFB 494. This research has made use of NASA Astrophysics Data System.

\section{References}

Aumann, H. H., Fowler, J. W., \& Melnyk, M. 1990, AJ, 99, 1674

Bensch, F., Stutzki, J., \& Ossenkopf, V. 2001, A\&A, 366, 636

Bensch, F., Leuenhagen, U., Stutzki, J., \& Schieder, R. 2003, ApJ, 591, 1013

Beuther, H., Kramer, C., Deiss, B., \& Stutzki, J. 2000, A\&A, 362, 1109

Cernicharo, J. 1985, ATM: A program to compute theoretical atmospheric opacity for frequency $<1000 \mathrm{GHz}$, Tech. Rep., IRAM

Cubick, M. 2005, The contribution of photon dominated regions to the farinfrared emission of the Milky Way: construction of PDR models, Diploma thesis, Universität zu Köln

Cubick, M., Röllig, M., Ossenkopf, V., Kramer, C., \& Stutzki, J. 2008, A\&A, submitted

Dame, T. M., Hartmann, D., \& Thaddeus, P. 2001, ApJ, 547, 792

de Jager, C., \& Nieuwenhuijzen, H. 1987, A\&A, 177, 217

Draine, B. T. 1978, ApJS, 36, 595

Draine, B. T., \& Bertoldi, F. 1996, ApJ, 468, 269

Eislöffel, J., Froebrich, D., Stanke, T., \& McCaughrean, M. J. 2003, ApJ, 595, 259

Falgarone, E., \& Phillips, T. G. 1996, ApJ, 472, 191

Fiege, J. D., \& Pudritz, R. E. 2000, MNRAS, 311, 105

Frerking, M. A., Langer, W. D., \& Wilson, R. W. 1982, ApJ, 262, 590

Gehman, C. S., Adams, F. C., \& Watkins, R. 1996, ApJ, 472, 673

Graf, U. U., Heyminck, S., Michael, E. A., et al. 2002, in Millimeter and Submillimeter Detectors for Astronomy, ed. T. G. Phillips, \& J. Zmuidzinas, Proc. SPIE, Hawaii/USA, 4855, 99, 167

Heithausen, A., Bensch, F., Stutzki, J., Falgarone, E., \& Panis, J. F. 1998, A\&A, 331,65

Herbig, G. H. 1998, AJ, 497, 736

Heyer, M. H., Brunt, C., Snell, R. L., et al. 1998, ApJS, 115, 241

Hiyama, S. 1998, Seitenbandkalibration radioastonomischer

Linienbeobachtungen, Diploma thesis, Universität zu Köln

Hollenbach, D. J., \& Tielens, A. G. G. M. 1999, Rev. Mod. Phys., 71, 173

Horn, J., Siebertz, O., Schmülling, F. et al. 1999, Exp. Astron., 9, 17

Howe, J. E., Jaffe, D. T., Genzel, R., \& Stacey, G. J. 1991, ApJ, 373, 158

Israel, F. P., \& Baas, F. 2002, A\&A, 383, 82

Jørgensen, J. K., Johnstone, D., Kirk, H., \& Myers, P. C. 2007, ApJ, 656, 293

Kaufman, M., Wolfire, M., Hollenbach, D., \& Luhman, M. 1999, ApJ, 527, 795

Klessen, R. S., \& Burkert, A. 2000, ApJS, 128, 287

Kramer, C., Stutzki, J., \& Winnewisser, G. 1996, A\&A, 307, 915 
Kramer, C., Degiacomi, C., Graf, U., et al. 1998a, in Advanced Technology MMV, Radio, and Terahertz Telescopes, ed. T. Phillips, Proc. SPIE, Kona/Hawaii/USA, 3357, 71

Kramer, C., Stutzki, J., Röhrig, R., \& Corneliussen, U. 1998b, A\&A, 329, 249

Kramer, C., Jakob, H., Mookerjea, B., et al. 2004, A\&A, 424, 887

Kramer, C., Mookerjea, B., Bayet, E., et al. 2005, A\&A, 441, 961

Kramer, C., Cubick, M., Röllig, M., et al. 2008, A\&A, 477, 547

Lada, E. A., \& Lada, C. J. 1995, AJ, 109, 1682

Lada, C. J., Muench, A. A., \& Luhman, K. L., et al. 2006, AJ, 131, 1574

Langer, W. D., \& Penzias, A. A. 1990, ApJ, 357, 477

Little, L. T., Gibb, A. G., Heaton, B. D., Ellison, B. N., \& Claude, S. M. X. 1994, MNRAS, 271, 649

Luhman, K. L., Rieke, G. H., Lada, C. J., \& Lada, E. A. 1998, ApJ, 508, 347

Luhman, K. L., Stauffer, J. R., \& Muench, A. A., et al. 2003, ApJ, 593, 1093

Luhman, K. L., Lada, E. A., Muench, A. A., \& Elston, R. J. 2005, ApJ, 618, 810

Meixner, M., \& Tielens, A. G. G. M. 1993, ApJ, 405, 216

Mookerjea, B., Kramer, C., Röllig, M., \& Masur, M. 2006, A\&A, 456, 235

Najita, J. R., Tiede, G. P., \& Carr, J. S. 2000, ApJ, 541, 977

Nakagawa, T., Yui, Y. Y., Doi, Y., et al. 1998, ApJS, 115, 259

Nakajima, Y., \& Hanawa, T. 1996, ApJ, 467, 321

Oka, T., Iwata, M., Maezawa, H., et al. 2004, ApJ, 602, 803

Ossenkopf, V., Röllig, M., Cubick, M., \& Stutzki, J. 2007, Molecules in Space

\& Laboratory, Paris, ed. J. L. Lemaire, \& F. Combes, 95
Ostriker, E. C., Stone, J. M., \& Gammie, C. F. 2001, ApJ, 546, 980 Padoan, P., Juvela, M., Bally, J., \& Nordlund, A\&A, 1998, ApJ, 504, 300 Plume, R., Bensch, F., Howe, J. E., et al. 2000, ApJ, 539, L133 Preibisch, T., \& Zinnecker, H. 2001, AJ, 122, 866 Preibisch, T., Zinnecker, H., \& Herbig, G. H. 1996, A\&A, 310, 456 Ridge, N. A., Wilson, T. L., Megeath, S. T., Allen, L. E., \& Myers, P. C. 2003, AJ, 126, 286

Röllig, M., Ossenkopf, V., Jeyakumar, S., \& Stutzki, J. 2006, A\&A, 451, 917

Röllig, M., Abel, N. P., Bell, T., et al. 2007, A\&A, 467, 187

Schneider, N., Simon, R., Kramer, C., et al. 2003, A\&A, 406, 915

Schröder, K., Staemmler, V., Smith, M. D., Flower, D. R., \& Jaquet, R. 1991, J. Phys. B, 24, 2487

Stacey, G. J., Jaffe, D. T., Geis, N., et al. 1993, ApJ, 404, 219

Stutzki, J., Stacey, G. J., Genzel, R., et al. 1988, ApJ, 332, 379

Stutzki, J., Bensch, F., Heithausen, A., Ossenkopf, V., \& Zielinsky, M. 1998, A\&A, 336, 697

Störzer, H., Stutzki, J., \& Sternberg, A. 1996, A\&A, 310, 592

Störzer, H., Stutzki, J., \& Sternberg, A. 1997, A\&A, 323, 13

Sun, K., Kramer, C., Ossenkopf, V., et al. 2006, A\&A, 451, 539

Tafalla, M., Kumar, M. S. N., \& Bachiller, R. 2006, A\&A, 456, 179

Winnewisser, G., Bester, M., \& Ewald, R. 1986, A\&A, 167, 207 